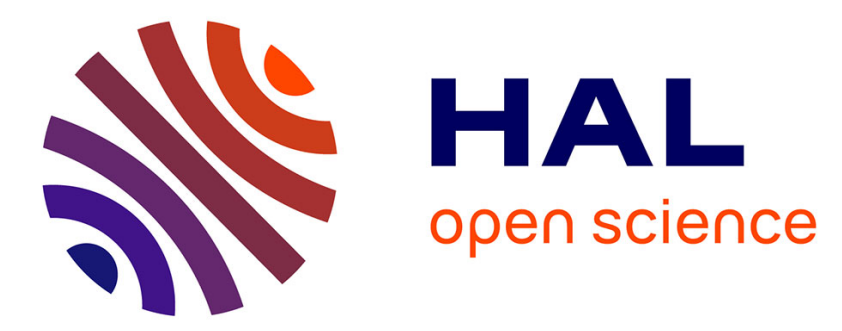

\title{
Performance validation for MMSE turbo equalization in ST-BICM systems
}

\author{
Nicolas Le Josse, Christophe Laot, Karine Amis Cavalec
}

\section{To cite this version:}

Nicolas Le Josse, Christophe Laot, Karine Amis Cavalec. Performance validation for MMSE turbo equalization in ST-BICM systems. VTC 2006 Fall: 64th IEEE Vehicular Technology Conference, September 25-28, Montreal, Canada, May 2006, Montreal, Canada. hal-02122808

\section{HAL Id: hal-02122808 \\ https://hal.science/hal-02122808}

Submitted on 11 Jun 2021

HAL is a multi-disciplinary open access archive for the deposit and dissemination of scientific research documents, whether they are published or not. The documents may come from teaching and research institutions in France or abroad, or from public or private research centers.
L'archive ouverte pluridisciplinaire $\mathbf{H A L}$, est destinée au dépôt et à la diffusion de documents scientifiques de niveau recherche, publiés ou non, émanant des établissements d'enseignement et de recherche français ou étrangers, des laboratoires publics ou privés. 


\title{
Performance Validation for MMSE Turbo Equalization in ST-BICM Systems
}

\author{
Nicolas Le Josse, Christophe Laot and Karine Amis \\ GET/ENST Bretagne, Dept. of Signal and Communications, TAMCIC (UMR CNRS 2872) \\ Technopôle de Brest Iroise, CS 8381829238 BREST Cedex \\ Email: nicolas.lejosse@enst-bretagne.fr, christophe.laot@enst-bretagne.fr, karine.amis@enst-bretagne.fr
}

\begin{abstract}
This paper presents a simple approach to assess performance that can be achieved by the MMSE turbo equalizer in ST-BICM systems over multipaths Rayleigh block fading channels with i.i.d fading statistics. By considering perfect information exchange between the SISO decoder and the linear MMSE equalizer, the performance reaches the matched filter bound, and a closed form expression of the corresponding probability of bit error can be derived at the output of the equalizer. In particular, we emphasize that the suggested approach provides an attractive and reliable tool for performance validation consistently with the proposed expression of the probability of bit error. Simulations for 4-PSK and 8-PSK modulated signals show the relevance of the proposed approach and the full benefit provided by the MMSE turbo equalizer. In addition some clarification of the signal-tonoise ratio definition is pointed out.
\end{abstract}

Index Terms-Space-Time BICM, MMSE Turbo equalization, performance validation, Rayleigh block fading multipath channel, probability of bit error expression.

\section{INTRODUCTION}

Space time bit interleaved coded modulation is used in multiple transmit and receive antenna systems [1][2] to increase data rates. Information bits are first encoded before being interleaved. Interleaved coded bits are splitted into $n_{T}$ streams and then passed through a modulator. Each of the resulting symbols is transmitted from an antenna. At the receiver, the MMSE turbo equalizer consists of a minimum mean square error (MMSE) equalizer and a decoder. The equalizer, with a priori information, performs iterative parallel soft interference cancellation and linear MMSE filtering. The result is then provided to the soft-input soft-output (SISO) decoder. Iterative information exchange on the coded bits is done between the two functions according to the MMSE turbo equalization principle [3][4][5].

The bit error rate (BER) at the output of the channel decoder is generally used to estimate the system performance. However, the complexity involved to obtain an exact expression of the probability of bit error after an iterative processing becomes quite high or in many cases prohibitive, and approximate bounds derived under various asymptotic conditions are generally used [6][2][7].

Instead of evaluating the BER at the output of the channel decoder, we consider the BER at the output of the MMSE equalizer. We derive the exact expression of the probability of bit error by using perfect information from the SISO decoder in the equalizer. We show that the proposed approach enables to accurately predict asymptotic performance and diversity improvement for the understanding of ST-BICM systems. In addition, this paper is motivated by the observation that performance comparison, when considering several papers, often results in a failure. Therefore, we suggest some clarification on the signal-to-noise ratio (SNR) definition to ensure reproducible results .

\section{MIMO DATA MODEL AND ASSUMPTIONS}

Consider the case of a MIMO multipaths Rayleigh block fading channel with $n_{T}$ transmit and $n_{R}$ receive antennas. As illustrated in Fig. 1, information bits are first encoded before being interleaved. Interleaved coded bits are serial to parallel converted and then modulated. Each of the $n_{T}$ resulting symbol streams is sent from one of the $n_{T}$ transmit antennas. We denote $s_{m}(d)$ the $d^{t h}$ symbol transmitted from the $m^{t h}$ transmit antenna. The transmitted symbols are assumed to be independent, identically distributed (i.i.d) and of variance $\sigma_{s}^{2}$. The modulation size is denoted $M$ and $Q$ is defined as $Q=\log _{2}(M)$.

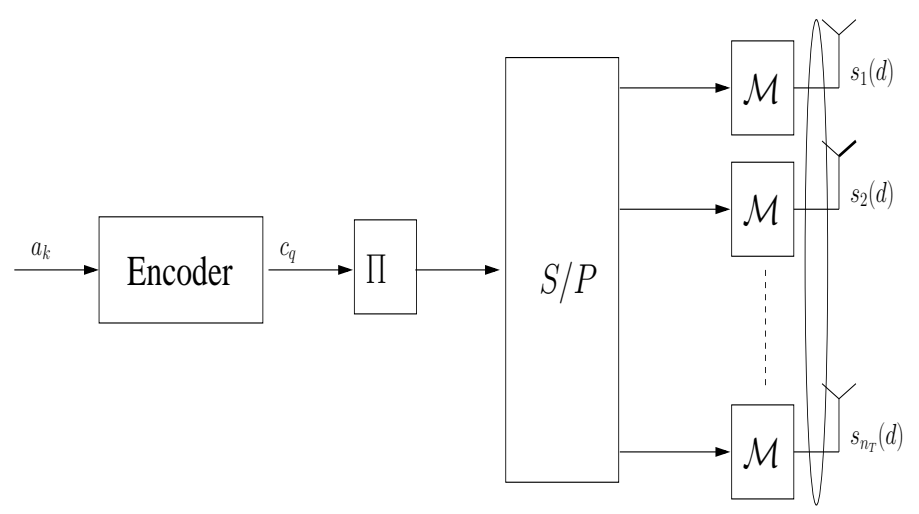

Fig. 1. ST-BICM transmission structure.

The transmitted symbols constructed as illustrated in Fig. 1 are organized as blocks of $N$ transmitted symbols on each antenna. Consecutive blocks of $N$ transmitted symbols are separated by a guard interval, which prevents from inter-block interference. Each guard interval is assumed to contain $L-1$ zeros (Zero-Padding). We assume a coherent symbol-spaced receiver with perfect carrier and time synchronization such

This work was supported in part by FT RD. 
that the samples received $y_{n}(d)$ at time $d$ on receive antenna $n$ can be represented by a discrete-time baseband model having $s_{m}(d)$ as input, corrupted by additive complex Gaussian noise samples $b_{n}(d)$ i.i.d, of zero-mean and variance $\sigma_{b}^{2}$

$$
y_{n}(d)=\sum_{l=0}^{L-1} \sum_{m=1}^{n_{T}} h_{n, m}(l) s_{m}(d-l)+b_{n}(d) n=1, . ., n_{R}
$$

where the discrete linear filter $h_{n, m}(l)_{l=0, \ldots, L-1}$ of length $L$ denotes the discrete-time equivalent channel model between transmit antenna $m$ and receive antenna $n$. The coefficients $h_{n, m}(l)_{l=0, . ., L-1}$ are assumed to be complex Rayleighdistributed, i.i.d, of zero mean and satisfying the power normalization constraint

$$
\mathrm{E}\left[\sum_{l=0}^{L-1}\left|h_{n, m}(l)\right|^{2}\right]=1
$$

Specifically, through the use of such Rayleigh block fading propagation channel and zero-padded transmission, we represent a communication model where transmitted blocks may result from frequency-hopping in Time Division Multiple Access (TDMA).

By grouping the received samples from all $n_{R}$ receive antennas at symbol time $d$, let us now introduce the $n_{R} \times 1$ channel output column vector $\mathbf{y}(d) \equiv\left\{y_{n}(d)\right\}_{n=1, \ldots, n_{R}}$ and the $n_{R} \times 1$ column vector of additive complexe noise samples $\mathbf{b}(d) \equiv\left\{b_{n}(d)\right\}_{n=1, \ldots, n_{R}}$. The corresponding mapped symbols of all transmit antennas at symbol time $d$ are given as a $n_{T} \times 1$ vector $\mathbf{s}(d) \equiv\left\{s_{m}(d)\right\}_{m=1, . ., n_{T}}$. For the equalizer derivation, it is convenient to consider the equivalent length $N_{F}=N_{F_{1}}+N_{F_{2}}+1$ sliding window model for the received signal. Over a block of $N_{F}$ symbol periods, we can relate $\mathbf{y}(d+k), k=0, . ., N_{F}-1$ to the corresponding vector of input samples as follows

$$
\underline{\mathbf{y}}(d)=\underline{\mathbf{H s}}(d)+\underline{\mathbf{b}}(d)
$$

where $\underline{\mathbf{y}}(d), \underline{\mathbf{s}}(d), \underline{\mathbf{b}}(d)$ are stacked vectors such that

$$
\underline{\mathbf{y}}(d)=\left[\begin{array}{lll}
\mathbf{y}(d)^{T} & \ldots & \mathbf{y}\left(d+N_{F}-1\right)^{T}
\end{array}\right]^{T}
$$

of dimension $n_{R} N_{F} \times 1$

$$
\underline{\mathbf{b}}(d)=\left[\begin{array}{lll}
\mathbf{b}(d)^{T} & \ldots & \mathbf{b}\left(d+N_{F}-1\right)^{T}
\end{array}\right]^{T}
$$

of dimension $n_{R} N_{F} \times 1$

$$
\underline{\mathbf{s}}(d)=\left[\begin{array}{lll}
\mathbf{s}\left(d-N_{F_{1}}\right)^{T} & \ldots & \mathbf{s}\left(d+N_{F_{2}}+L-1\right)^{T}
\end{array}\right]^{T}
$$

of dimension $n_{T}\left(N_{F}+L-1\right) \times 1$ and $\underline{\mathbf{H}}$ is a block-Toeplitz channel matrix

$\underline{\mathbf{H}}=\left[\begin{array}{ccccc}\mathbf{H}(0) & \ldots & \mathbf{H}(L-1) & 0 & \ldots \\ 0 & \mathbf{H}(0) & \ldots & \mathbf{H}(L-1) & 0 \\ \vdots & \ddots & \ddots & \ddots & \vdots \\ 0 & \vdots & \mathbf{H}(0) & \ldots & \mathbf{H}(L-1)\end{array}\right]$ of dimension $n_{R} N_{F} \times n_{T}\left(N_{F}+L-1\right)$ consisting of blocks $\mathbf{H}(l)$ given by

$$
\mathbf{H}(l)_{l=1, . ., L}=\left[\begin{array}{ccc}
h_{1,1}(l) & \ldots & h_{1, n_{T}}(l) \\
\vdots & \ddots & \vdots \\
h_{n_{R}, 1}(l) & \ldots & h_{n_{R}, n_{T}}(l)
\end{array}\right]
$$

of dimension $n_{R} \times n_{T}$.

\section{AVERAGE SNR PER ANTENNA DEFINITION}

The transmitted symbols from each antenna have an equal variance of $\sigma_{s}^{2}=P / n_{T}$ so that the total transmitted power is constrained to $P$. The MIMO channel relates the average signal power to noise power per receive antenna $n$ according to:

$$
\bar{\gamma}=\sum_{l=0}^{L-1} \sum_{m=1}^{n_{T}} \mathrm{E}\left[\left|h_{n, m}(l)\right|^{2}\right] \sigma_{s}^{2} / \sigma_{b}^{2}=P / \sigma_{b}^{2}
$$

where we assume the following normalization constraint on the fading coefficients: $\left.\mathrm{E}\left[\left|h_{n, m}(l)\right|^{2}\right\}\right]_{\forall n, m, l}=1 / L$.

When considering the signal to noise ratio $\bar{\gamma}$ measured in a symbol rate bandwidth $1 / T_{s}$ : each receive antenna collects a total power of $E_{b} D_{b}$ with a corresponding noise power $N_{0} / T_{s}$, where $E_{b}$ is the average transmitted bit energy, $D_{b}$ the transmitted bit rate and $N_{0}$ the noise power spectral density. Assuming a channel code rate $R$ and a constellation order $M$, the transmitted bit rate is $D_{b}=R n_{T} \log _{2}(M) / T_{s}$ bits/s. If the reception low-pass filter is the square root of a raised cosinus filter and under the ideal Nyquist band-limited filtering assumption, the average gaussian noise power is equal to $P_{b}=N_{O} / T_{S}$. Therefore the relationship between the average signal to noise ratio per receive antenna $\bar{\gamma}$ and the signal to noise ratio per information bit $E_{b} / N_{0}$ is given by

$$
\bar{\gamma}=E_{b} D_{b} /\left(N_{0} / T_{s}\right)=R n_{T} \log _{2}(\mathrm{M}) E_{b} / N_{0}
$$

\section{TURbo EQUALIZATION OF ST-BICM SYSTEM}

The structure of the investigated iterative receiver is shown in Fig. 2. The receiver consists of the concatenation of a Soft Input / Soft Output equalizer and a Soft Input / Soft Output decoder exchanging information on the coded bits in an iterative manner according to the MMSE turbo equalization principle [3]. Here is the main turbo equalization principle algorithm. The detailed algorithm can be found in [4][5]. Based on the interleaved a posteriori probabilities about all coded bits available at the decoder output, the detector calculates the soft symbol values for both multiantenna interference (MAI) and intersymbol interference (ISI). If $\mathrm{L}_{\mathrm{IN}_{m}}^{\mathrm{E}}(q)$ denotes the Logarithm of Likelihood Ratio (LLR) for the $q$ th encoded bit from $m$ th transmit antenna (i.e after interleaver and serial to parallel conversion), the soft symbol values $\tilde{s}_{m}(d)$ are computed taking the conditional expectation $\mathrm{E}\left[s_{m}(d) \mid\left\{\mathrm{L}_{\mathrm{IN}_{m}}^{\mathrm{E}}(q)\right\}_{Q d \leq q \leq Q(d+1)-1}\right]$. If $\left\{c_{q}\right\}_{Q d \leq q \leq Q(d+1)-1}$ is refered as binary components corresponding to the complex signal set $\mathcal{M}$, we can write

$$
\tilde{s}_{m}(d)=\sum_{s \in \mathcal{M}, c_{q} \leftrightarrow s} s \prod_{q=Q d}^{Q(d+1)-1} \mathrm{P}\left(c_{q} \mid\left\{\mathrm{L}_{\mathrm{IN}_{m}}^{\mathrm{E}}(q)\right\}\right)
$$




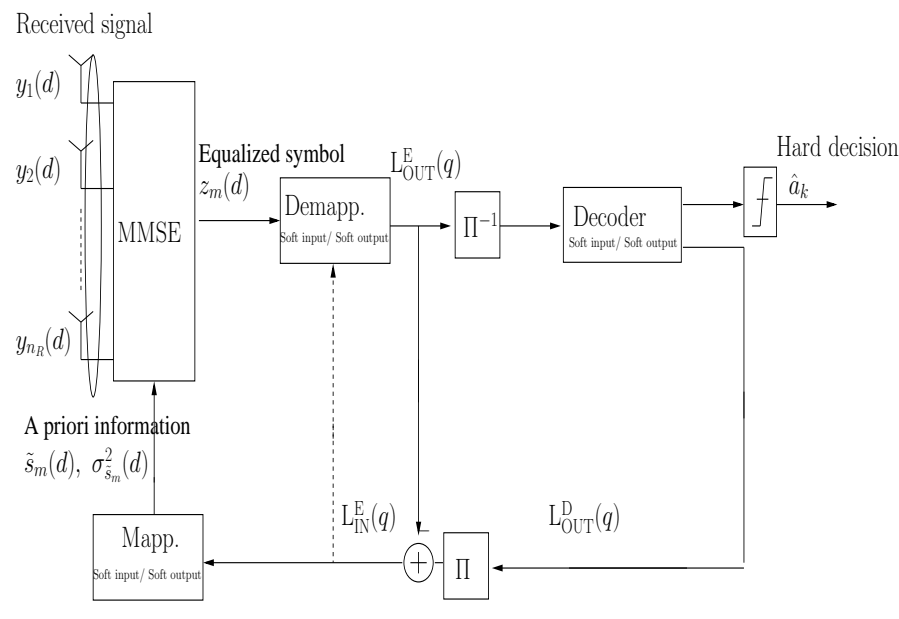

Fig. 2. MMSE Turbo equalizer structure

and the corresponding variance

$$
\sigma_{\tilde{s}_{m}(d)}^{2}=\sum_{s \in \mathcal{M}, c_{q} \leftrightarrow s}|s|^{2} \prod_{q=Q d}^{Q(d+1)-1} \mathrm{P}\left(c_{q} \mid\left\{\mathrm{L}_{\mathrm{IN}_{m}}^{\mathrm{E}}(q)\right\}\right)
$$

where $\mathrm{P}\left(c_{q} \mid\left\{\mathrm{L}_{\mathrm{IN}_{m}}^{\mathrm{D}}(q)\right\}\right)$ can be computed as $\frac{1}{2}(1+$ $\left.\left(2 c_{q}-1\right) \tanh \left(\frac{\mathrm{L}_{\mathrm{IN} m}^{\mathrm{E}}(q)}{2}\right)\right)$ [8].

Using these data estimates and the channel coefficients, a soft replica of the interference is computed for each of the $n_{T}$ antennas, and then substracted from the total received signal $\underline{\mathbf{y}}(d)$. Let introduce the $n_{T}\left(N_{F}+L-1\right) \times 1$ dimensional vector $\underline{\tilde{\mathbf{y}}}_{\mathbf{m}}(d)=\underline{\mathbf{y}}(d)-\underline{\mathbf{H}} \tilde{\mathbf{s}}(d)$ of residual interference for all $m=1, \ldots, n_{T}$, where $\underline{\tilde{\mathbf{s}}}(d)$ denotes the $n_{T}\left(N_{F}+L-1\right) \times 1$ dimensional vector of soft symbol values. We follow the approach described in [9] and represent the MMSE filter input signal as the desired component added to the residual interference

$$
\underline{\tilde{\mathbf{r}}}_{\mathbf{m}}(d)=\underline{\mathbf{h}}_{\boldsymbol{\Delta}_{\mathbf{m}}} \tilde{s}_{m}(d)+\underline{\tilde{\mathbf{y}}}_{\mathbf{m}}(d)
$$

where $\underline{\mathbf{h}}_{\Delta_{m}}$ is the $\Delta_{m}^{t h}$ column vector of channel matrix $\underline{\mathbf{H}}$ corresponding to the different fadings experienced by the desired component. The index $\Delta_{m}$ is the decision delay parameter required in the derivation of the equalizer transfer function such that $\Delta_{m}=N_{F_{1}}+1$.

The remaining signal is processed by the MMSE filter to further suppress the residual interference. The $n_{R} N_{F} \times 1$ dimensional filter $\underline{\mathbf{w}}_{\mathbf{m}}(d)$ wich minimizes the mean square error between the input $\tilde{\underline{\mathbf{r}}}_{\mathbf{m}}(d)$ and the estimated symbol $s_{m}(d)$

$$
\mathrm{E}\left[\left|\underline{\mathbf{w}}_{\mathbf{m}}{ }^{H}(d) \underline{\tilde{\mathbf{r}}}_{\mathbf{m}}(d)-s_{m}(d)\right|^{2} \mid \underline{\tilde{\mathbf{s}}}(d)\right]
$$

provides us with the following equalized symbol for each index $m=1, . ., n_{T}$

$$
z_{m}(d)=\mu_{m}(d) \tilde{s}_{m}(d)+\underline{\mathbf{w}}_{\mathbf{m}}{ }^{H}(d) \underline{\tilde{\mathbf{y}}}_{\mathbf{m}}(d)
$$

where

$$
\begin{aligned}
& \underline{\mathbf{w}}_{\mathbf{m}}{ }^{H}(d)=\alpha_{m}(d) \sigma_{s}^{2} \underline{\boldsymbol{\Sigma}}(d)^{-1} \underline{\mathbf{h}}_{\Delta_{m}}^{H} \\
& \mu_{m}(d)=\underline{\mathbf{w}}_{\mathbf{m}}(d)^{H} \underline{\mathbf{h}}_{\boldsymbol{\Delta}_{\mathbf{m}}}
\end{aligned}
$$

in which the $n_{R} N_{F} \times n_{R} N_{F}$ covariance matrix of the residual interference in (13) is given by

$$
\underline{\boldsymbol{\Sigma}}(d)=\underline{\mathbf{H} \boldsymbol{\Lambda}}(d) \underline{\mathbf{H}}^{H}+\sigma_{b}^{2} \mathbf{I}_{N_{F} n_{R}}
$$

and is common to all $m$. The $n_{T}\left(N_{F}+L-1\right) \times n_{T}\left(N_{F}+L-1\right)$ diagonal matrix $\underline{\Lambda}(d)$ denotes the error covariance matrix of the soft symbol estimates obtained as $\operatorname{Diag}(\mathrm{E}[\mid \underline{\mathbf{s}}(d)-$ $\left.\left.\left.\underline{\tilde{\mathbf{s}}}(d)\right|^{2} \mid \underline{\tilde{\mathbf{s}}}(d)\right]\right)$. The intermediate variables $\alpha_{m}(d)$ are computed as

$$
\alpha_{m}(d)=\frac{1}{1+\sigma_{\tilde{s}}^{2}(d) \underline{\mathbf{h}}_{\boldsymbol{\Delta}_{\mathbf{m}}}{ }^{H} \underline{\boldsymbol{\Sigma}}(d)^{-1} \underline{\mathbf{h}}_{\boldsymbol{\Delta}_{\mathbf{m}}}}
$$

Since the elements of $\underline{\boldsymbol{\Lambda}}(d)$ are not constant, the MMSE solution is time-variant and (16) have to be computed for each index $d$.

The detector generates soft estimates of the coded bits conditional on the equalizer output $z_{m}(d)$, as well as a priori information about all coded bits. Assuming that the LLR $\mathrm{L}_{\mathrm{OUT}_{m}}^{\mathrm{E}}(q)$ refered as binary components to symbol $s_{m}(d)$ at time $d$ defined as $q \bmod (Q)+1$, we can write

$$
\mathrm{L}_{\mathrm{OUT}_{m}}^{\mathrm{E}}(q)=\ln \frac{\mathrm{P}\left(c_{q}=1 \mid z_{m}(d)\right)}{\mathrm{P}\left(c_{q}=0 \mid z_{m}(d)\right)}-\mathrm{L}_{\mathrm{IN}_{m}}^{\mathrm{E}}(q)
$$

where the a priori LLR $\mathrm{L}_{\mathrm{IN}_{m}}^{\mathrm{E}}(q)$ fed back from the decoder is substracted out since equalizer should deliver extrinsic information only. The above expression is computed by using the gaussian assumption for the term of residual interference in (15) as in [5]. Given the observation and prior probabilities after deinterleaving and parallel to serial conversion, the decoder computes the marginal log-likelihood of the coded bits in the form $\mathrm{L}_{\mathrm{OUT}}^{\mathrm{D}}\left(c_{q}\right)$ [10]. At the last iteration, the decoder provides hard decisions $\hat{a}_{k}$ on information bits.

\section{Performance Validation for mMSE Turbo EQUALIZATION: PROPOSED APPROACH}

First of all, we assume a block fading environment and perfect a priori information feeding the filter (16) (i.e $\underline{\boldsymbol{\Lambda}}(d)=\mathbf{0}$ in (17)). The so-called genie MMSE equalizer suppresses multiantenna interference and intersymbol interference components for each transmit antenna, from the received signal. We can write the output of the equalizer as an estimate of $s_{m}(d)(15)$

$$
z_{m}(d)=\frac{\sigma_{s}^{2}\left\|\underline{\mathbf{h}}_{\boldsymbol{\Delta}_{\mathbf{m}}}\right\|^{2}}{\sigma_{s}^{2}\left\|\underline{\mathbf{h}}_{\boldsymbol{\Delta}_{\mathbf{m}}}\right\|^{2}+\sigma_{b}^{2}} s_{m}(d)+\frac{\sigma_{s}^{2} \underline{\mathbf{h}}_{\boldsymbol{\Delta}_{\mathbf{m}}}{ }^{H}}{\sigma_{s}^{2}\left\|\underline{\mathbf{h}}_{\boldsymbol{\Delta}_{\mathbf{m}}}\right\|^{2}+\sigma_{b}^{2}} \underline{\mathbf{b}}(d)
$$

In that case we can consider the estimated output symbol from each transmit antenna $m$ as the output of an equivalent ISI+MAI free AWGN channel. Let us now write the average SNR computed over different blocks at the output of the equalizer as a function of $\bar{\gamma}$ defined in (10). It is easily shown that $\mathrm{SNR}_{m}=\left\|\underline{\mathbf{h}}_{\boldsymbol{\Delta}_{\mathrm{m}}}\right\|^{2} \bar{\gamma}$. By considering the Toeplitz structure of the channel matrix $\underline{\mathbf{H}}$ (7), let us write $\left\|\underline{\mathbf{h}}_{\boldsymbol{\Delta}_{\mathbf{m}}}\right\|^{2}$ as $\kappa=$ $\left(\sum_{k=0}^{n_{R} L-1} \kappa_{k}^{2}\right)$ where the channel coefficients $\left\{\kappa_{k}\right\}_{k=1, \ldots, n_{R} L}$ are i.i.d complex gaussian with zero mean. Therefore, $\kappa$ has a chi-squared distribution with $2 n_{R} L$ degrees of freedom and variance $1 / L$. Assuming soft demapping without a priori 
knowledge, the conditional probability of bit error for MPSK modulated signals in time-invariant environment with perfect CSI at the receiver, can be expressed as: $\mathrm{P}_{\mathrm{e} \kappa}=$ $\mathrm{P}_{\text {eM-PSK }}(\kappa \bar{\gamma})$. Hence, to find the probability of error in a block fading environment, we average the above BER over the fading channel statistics of $\kappa$.

In case of 4-PSK modulated signals, the conditional probability of bit error for M-PSK can be expressed as [11]: $\mathrm{P}_{\mathrm{e} 4-\mathrm{PSK}}=\mathrm{E}_{\kappa}\left[\frac{1}{2} \operatorname{erfc}\left(\sqrt{\kappa \frac{\bar{\gamma}}{2}}\right)\right]$. There is a closed-form solution as

$$
\begin{aligned}
& \mathrm{P}_{\mathrm{e} 4-\mathrm{PSK}}=\left[\frac{1}{2}\left(1-\sqrt{\frac{\gamma / L}{1+\gamma / L}}\right)\right]^{L n_{R}} \\
& \sum_{k=0}^{L n_{R}-1}\left(\begin{array}{c}
L n_{R}-1+k \\
k
\end{array}\right)\left[\frac{1}{2}\left(1+\sqrt{\frac{\gamma / L}{1+\gamma / L}}\right)\right]^{k}
\end{aligned}
$$

in which we define $\bar{\gamma}=n_{T} \log _{2}(M) \gamma$. Note that this result is identical to the average probability of error for a maximum ratio combining scheme over a flat fading channel [11] with diversity of order $n_{R} L$. Hence our system benefits from a diversity of order $L$ due to the channel dispersion in addition to the explicit $n_{R}$ order spatial diversity. This also coincides with the matched filter bound in ST-BICM systems at the output of the genie MMSE equalizer.

Expression for higher order signaling 8-PSK mapping can be similarly derived [12]

$$
\begin{aligned}
& \mathrm{P}_{\text {e8-PSK }}=\frac{1}{2}- \\
& \sum_{j=1}^{2} \frac{1}{3} \vartheta_{j}\left\{\left(\frac{1}{2}+\frac{\arctan \left(\varrho_{j}\right)}{\pi}\right) \sum_{k=0}^{n_{R} L-1}\left(\begin{array}{c}
2 k \\
k
\end{array}\right) \frac{1}{\left[4\left(\varsigma_{j}^{2}+1\right)\right]^{k}}\right. \\
& \left.+\frac{\sin \left(\arctan \left(\varrho_{j}\right)\right)}{\pi} \sum_{k=1}^{n_{R} L-1} \sum_{i=1}^{k} \frac{T_{i k} \cos ^{2(k-i)+1}\left(\arctan \left(\varrho_{j}\right)\right)}{\left(\varsigma_{j}^{2}+1\right)^{k}}\right\}
\end{aligned}
$$

$$
\left\{\begin{array}{l}
\varsigma_{j}=\frac{3(\gamma / L)\left(\sqrt{2}+(-1)^{j}\right)}{2 \sqrt{2}} j=1,2 \\
\vartheta_{j}=\frac{3(\gamma / L)\left(\sqrt{2}+(-1)^{j}\right)}{3(\gamma / L)\left(\sqrt{2}+(-1)^{j}\right)+2 \sqrt{2}} j=1,2 \\
\varrho_{j}=\frac{3(\gamma / L)\left(\sqrt{2}-(-1)^{j}\right)}{3(\gamma / L)\left(\sqrt{2}+(-1)^{j}\right)+2 \sqrt{2}} j=1,2 \\
T_{i k}=\frac{\left(\begin{array}{c}
2 k \\
k
\end{array}\right)}{\left(\begin{array}{c}
2(k-i) \\
k-i
\end{array}\right) 4^{i}(2(k-i)+1)}
\end{array}\right.
$$

where we assume a bit-symbol Gray mapping. Note that, best asymptotic performance will be obtained when considering a priori information during the soft demapping process in (19) [13].

\section{RESUlTS AND DISCUSSION}

To evaluate the performance of the linear MMSE equalizer with a priori information, we consider a block transmission over different MIMO configurations with $n_{T}=n_{R}=2,4$ and perfect CSI at the receiver. The frame size is 512 information bits. Information bits are first encoded with a 64state convolutional of rate $1 / 3$ and code generator polynomials $(133,145,175)$. The coded bits are then interleaved by a pseudo-random permutation, serial to parallel converted and 4PSK or 8-PSK modulated. The channel is multipaths Rayleigh block fading with respectively $L=2$ and $L=10$ paths and variance $1 / L$. Guard intervals of length $L-1$ are inserted between successive blocks to prevent from interblock interference. In the MMSE equalizer, the filter coefficient number is set to $N_{F}=9\left(N_{F_{1}}=N_{F_{2}}=4\right)$ for $L=2$ and $\left(N_{F}=21\right.$ $\left.N_{F_{1}}=N_{F_{2}}=10\right)$ for $L=10$.

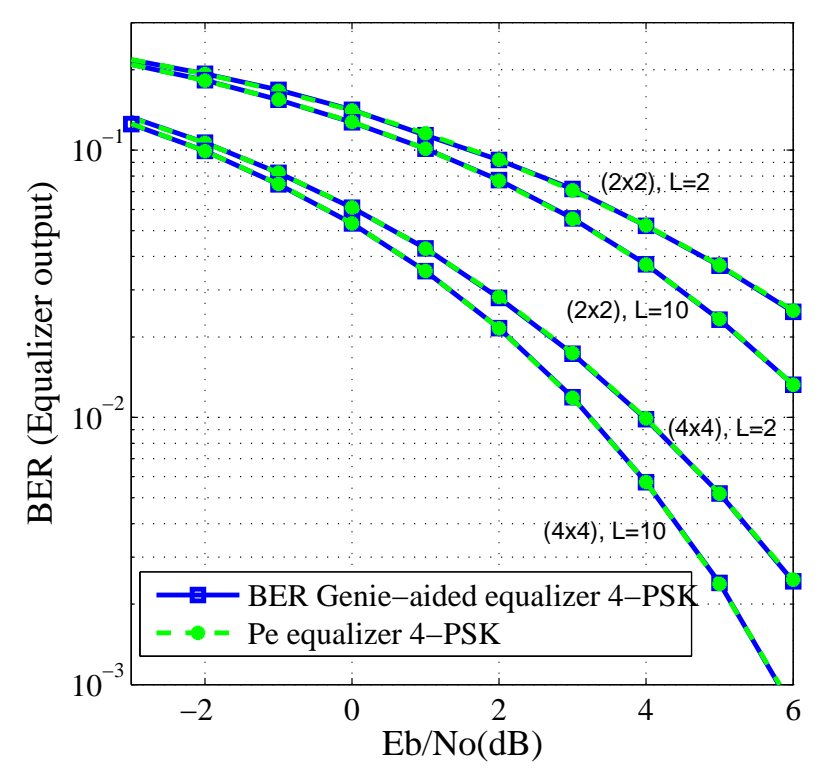

Fig. 3. Performance comparison of the MMSE equalizer with perfect a priori information and the corresponding probability of bit error expression in STBICM system with $n_{T}=n_{R}=2,4$ over Rayleigh block fading channel of order $L=2$ and $L=10$ for 4-PSK modulated signals. The performance of the MMSE equalizer with perfect a priori information perfectly matches that of the proposed probability of bit error expression. This demonstrates also the benefi ts offered by the considered equalizer to exploit multipaths.

In Fig. 3 and Fig. 4, we compare the bit error rate of the MMSE equalizer with perfect a priori information and the corresponding analytical probability of bit error developed in (21) and (22) respectively for 4-PSK and 8-PSK modulated signal. In each MIMO configuration, we observe that both curves are indistinguishable which confirms the relevance of the proposed BER expression. The figure also shows performance improvements due to the receive diversity with increasing number of receive antennas and order of channel 
impulse response length. The BER of the MMSE equalizer and the channel decoder with estimated a priori information for $n_{T}=n_{R}=2$ over multipaths Rayleigh block fading channel of order $L=2$ for 8-PSK modulated signal and 1-6 iterations is shown in Fig. 5. The BER of the MMSE equalizer is compared to the proposed probability of bit error expression as a reference. The iterative process reaches a steady-state which matches the analytical bound. The genie curves are also reported. The conclusion is that simulations confirm the purpose and provide a solid basis for assessing performance that can be achieved by the MMSE turbo equalization in STBICM systems.

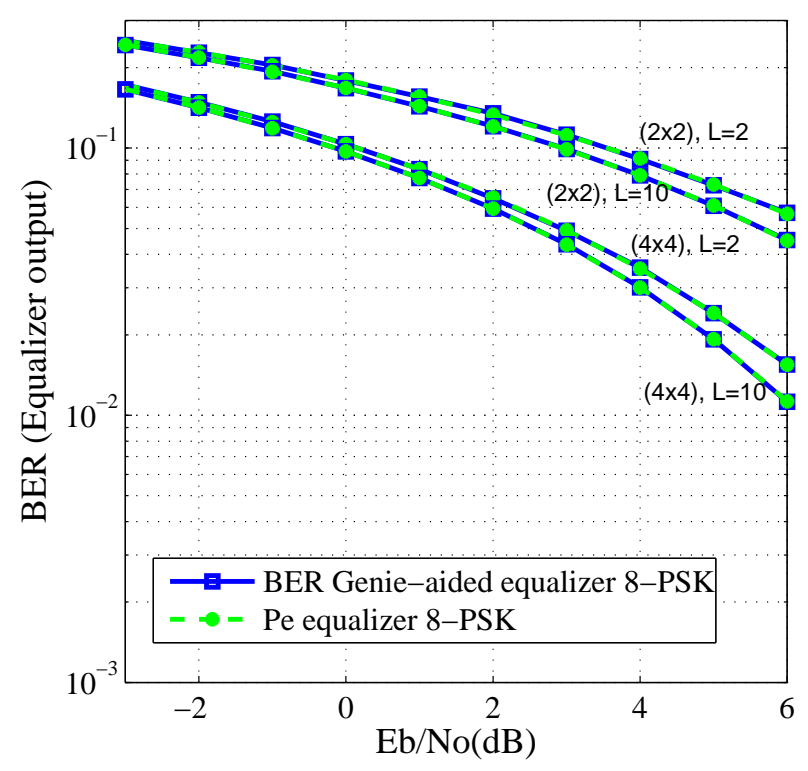

Fig. 4. Performance comparison of the MMSE equalizer with perfect a priori information and the corresponding probability of bit error expression in STBICM system with $n_{T}=n_{R}=2,4$ over Rayleigh block fading channel of order $L=2$ and $L=10$ for 8-PSK modulated signals. The performance of the MMSE equalizer with perfect a priori information perfectly matches that of the proposed probability of bit error expression. This demonstrates also the benefi ts offered by the considered equalizer to exploit multipaths.

\section{CONCLUSION}

A performance validation of the MMSE turbo equalizer in ST-BICM systems over multipaths Rayleigh block fading channels with i.i.d fading statistics has been proposed. By considering the output of the MMSE equalizer with perfect a priori information (genie), we have derived a closed form expression of the probability of bit error which resulted in a reliable tool for performance validation. The relevance of the proposed approach has been shown to be consistent with the results reported in our simulations for 4-PSK and 8-PSK modulated signals. Of course, expressions for higher order modulations can be similarly derived. Finally, we would like to emphasize that the proposed approach could be extended to find closed form expression of the probability of bit error over multipaths Rayleigh block fading channels with dissimilar i.i.d fading statistics.

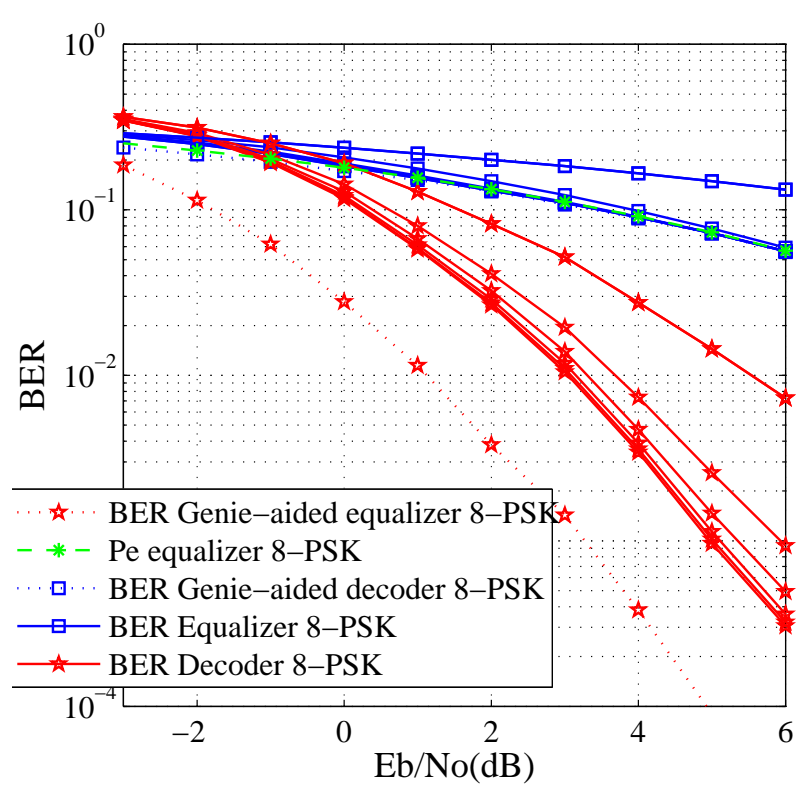

Fig. 5. Bit-error performance of the MMSE equalizer and the channel decoder in ST-BICM system with $n_{T}=n_{R}=2$ over Rayleigh block fading channel of order $L=2$ for 8-PSK modulated signal. 6 iterations are performed. Three iterations are necessary to the MMSE equalizer to reach the analytical probability of bit error.

\section{REFERENCES}

[1] S. Chtourou, R. Visoz, and A. O. Berthet, "A Class of Low Complexity Iterative Equalizers for Space-Time BICM over MIMO Block Fading Multipath AWGN Channel," Comm. 2004, IEEE International Conf., vol. 1, pp. 618-624, June. 2004.

[2] X. Wautelet, A. Dejonghe, and L. Vandendorpe, "MMSE-Based Turbo Receiver for Space-Time BICM Over Frequency-Selective MIMO Fading Channels," IEEE Trans. Signal Processing, vol. 52(6), pp.1804-1809, June. 2004.

[3] A. Glavieux, C. Laot, and J. Labat, "Turbo equalization over a frequency selective channel," Symp on Turbo-Codes, vol. pp.96-102, Sept. 1997.

[4] X. Wang and H. Poor, "Iterative (turbo) soft interference cancellation and decoding for coded CDMA," IEEE Trans. Commun., vol. 47(7), pp.1046-1061, July. 1999.

[5] M. Tüchler, A. Singer, and R. Koetter, "Minimum mean squared error equalization using a priori information," IEEE Trans. Signal processing, vol. 50(3), pp.673-683, Mar. 2002.

[6] V. Tarokh, N. Seshadri, and A. Calderbank, "Space-time Codes for High Data Rate Wireless Communication: Performance Criterion and Code Construction," IEEE Trans. Inform. Theory, vol. 17(3), pp.451-460, Mar. 1999.

[7] S. Brink, "Convergence of iterative decoding," Electron. Lett., vol. 35(13), pp. 1117-1119, June 1999.

[8] C. Laot, R. Le Bidan, and D. Leroux, "Low comlexity linear turbo equalization: Apossible solution for EDGE," IEEE Trans. Wireless. Commun., vol. 4(3), pp. 965-974, May 2005.

[9] K. Kansanen, C. Schneider, T. Matsumoto, and R. Thoma, "MultilevelCoded QAM with MIMO Turbo-Equalization in Broadband SingleCarrier Signaling," Vehicular Technology Conf.Proceeding,, vol. 54(3), pp.954-966, May. 2005.

[10] L. Balh, J. Cocke, F. Jelinek, and J. Raviv, "Optimal decoding of linear codes for minimizing symbol error rate," IEEE Trans. Inf. Theory, vol. pp.284-287, March 1974.

[11] J. Proakis, Digital Communications, McGraw Hill, Ed. 2nd Ed., 1995.

[12] S. Chennakeshu and J. Anderson, "Error rates for Rayleigh Fading Multichannel Reception of MPSK Signals," IEEE Trans. Commun., vol. 43(2), pp.338-346, Feb. 1995.

[13] S. Brink, J. Speidel, and R. Yan, "Iterative Demapping and Decoding for Multilevel Modulation," Proc. IEEE Global Telecommun. Conf. (GLOBECOM), Sydney, Australia, vol. pp.579-584, Nov. 1998. 